\title{
MicroRNA-223 Displays a Protective Role Against Cardiomyocyte Hypertrophy by Targeting Cardiac Troponin I-Interacting Kinase
}

\author{
Yao-Sheng Wanga Jing Zhou ${ }^{b}$ Kui Hong ${ }^{c}$ Xiao-Shu Cheng ${ }^{c}$ Yi-Gang Lia \\ aDepartment of Cardiology, XinHua Hospital Affiliated to Shanghai Jiao Tong University School of \\ Medicine, Shanghai, 'bShanghai Institute of Health Sciences, Shanghai, 'Department of Cardiology, \\ Second Affiliated Hospital, Nanchang University, Nanchang, PR China
}

\section{Key Words}

MicroRNA-223 • TNNI3K • Cardiomyocyte $\cdot$ Hypertrophy

\begin{abstract}
Background/Aims: MicroRNAs play regulatory role in cardiovascular disease. MicroRNA-223 (miR-223) was found to be expressed abundantly in myocardium. TNNI3K, a novel cardiac troponin I (cTnI)-interacting and cardiac hypertrophy related kinase, is computationally predicted as a potential target of miR-223. This study was designed to investigate the cellular and molecular effects of miR-223 on cardiomyoctye hypertrophy, focusing on the role of TNNI3K. Methods: Neonatal rat cardiomyocytes (CMs) were cultured, and CMs hypertrophy was induced by endothelin-1 (ET-1). In vivo cardiac hypertrophy was induced by transverse aorta constriction (TAC) in rats. Expression of miR-223 in CMs and myocardium was detected by real-time PCR (RT-PCR). MiR-223 and TNNI3K were overexpressed in CMs via chemically modified sense RNA (miR-223 mimic) transfection or recombinant adenovirus infection, respectively. Cell size was measured by surface area calculation using fluorescence microscopy after anti- $\alpha$-actinin staining. Expression of hypertrophy-related genes was detected by RTPCR. The protein expression of TNNI3K and CTnI was determined by Western blots. Luciferase assay was employed to confirm the direct binding of miR-223 to the 3'UTR of TNNI3K mRNA. Intracellular calcium was measured by sensitive fluorescent indicator (Furo-2). Video-based edge detection system was employed to measure cardiomyocyte contractility. Results: MiR223 was downregulated in ET-1 induced hypertrophic CMs and in hypertrophic myocardium compared with respective controls. MiR-223 overexpression in CMs alleviated ET- 1 induced hypertrophy, evidenced by smaller cell surface area and downregulated ANP, $\alpha$-actinin, Myh6 and Myh7 expression. Luciferase reporter gene assay showed that TNNI3K serves as a direct target gene of miR-223. In miR-223-overexpressed CMs, the protein expression of TNNI3K was significantly downregulated. MiR-223 overexpression also rescued the upregulated TNNI3K
\end{abstract}

Y.-S. Wang and J. Zhou contributed equally to this work.

Yi-Gang Li, M.D

KARGER 125
Department of Cardiology, XinHua Hospital affiliated to Shanghai Jiao Tong University School of Medicine, 1665 Kongjiang Street, Shanghai 200092 (PR China) E-Mail xinhuaxn@163.com. 


\section{Cellular Physiology Cell Physiol Biochem 2015;35:1546-1556

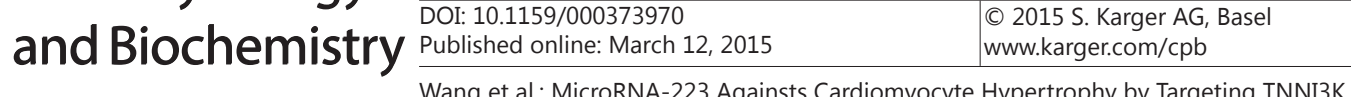

expression in hypertrophic CMs. Furthermore, cTnI phosphorylation was downregulated post miR-223 overexpression. Ad.rTNNI3K increased intracellular $\mathrm{Ca}^{2+}$ concentrations and cell shortening in CMs, while miR-223 overexpression significantly rescued these hypertrophic effects. Conclusion: By direct targeting TNNI3K, miR-223 could suppress CMs hypertrophy via downregulating cTnI phosphorylation, reducing intracellular $\mathrm{Ca}^{2+}$ and contractility of CMs. miR-223 / TNNI3K axis may thus be major players of CMs hypertrophy.

Copyright (c) 2015 S. Karger AG, Basel

\section{Introduction}

Cardiac hypertrophy is characterized by an increase in the size of individual cardiomyocyte. The pathologic process of cardiomyocyte hypertrophy is highly related to complex alteration in cardiac gene expression pattern, and changes in molecular pathways [1]. Elucidation of signaling cascades involved in cardiomyocyte hypertrophy will be of critical importance for the design of target molecular therapy against cardiac hypertrophy.

MicroRNAs (miRNAs) are small RNAs approximately 22 nucleotides in length that bind the $3^{\prime}$ untranslated region ( $3^{\prime}$-UTR) of messenger RNAs to post-transcriptionally regulate the translation of target genes [2]. Accumulating evidence suggests that miRNAs may play important roles in the pathology of cardiovascular system, especially in the cardiac hypertrophy $[3,4]$.

As one of miRNAs with multiple biologic functions, miR-223 has been studied widely in recent years. It is now clearly established that miR-223 plays an essential role in the proliferation of granulocytes, gastric cancer cells, venous smooth muscle cells, and hematopoietic cells [5-8], and it is also a key factor during osteoclast differentiation [9]. Recent report demonstrated a role for miR-223 in cardiac function and tissue metabolism [10], and in addition, circulating levels of miR-223 are responsive to therapeutic interventions and change during the progression of hypertension-induced heart disease [11]. These data attract special interest to researchers in the cardiovascular field and hint a potential role of miR-223 in pathologic process of cardiac hypertrophy. However, the mechanisms by which miR-223 regulates the biological characteristics of cardiomyocyte (CMs) hypertrophy are not fully understood.

Using bioinformatics predictions [12], complementary binding sites have been identified in both miR-223 and the $3^{\prime}$-UTR of the cardiac troponin I-interacting kinase (TNNI3K) mRNA. TNNI3K, also known as CARK, is a novel cardiac-specific kinase. It contains a central kinase domain, flanking by an ankyrin repeat domain in the amino terminus and a serine-rich domain in the carboxyl terminus [13]. Studies have revealed that TNNI3K is a functional kinase which could directly interact with cardiac troponin I (cTnI), and is actively involved in regulating cardiac remodeling [14]. TNNI3K induced CMs hypertrophy was already demonstrated in vitro studies [15]. Based on these data, we hypothesized that miR223 might act as an upstream regulator of TNNI3K, thereby modulatethe CMs hypertrophy process.

To confirm and further investigate the potential role of miR-223 / TNNI3K pathway in pathologic process of CMs hypertrophy, endothelin-1 (ET-1, a peptide hormone) was routinely employed to establish model of CMs hypertrophy in vitro [16], and expression of miR-223 was detected in ET-1 induced hypertrophic CMs. Myocardial expression of miR-223 was also detected in rats post transverse aorta constriction (TAC). In addition, the cellular and molecular effects of miR-223 overexpression on CMs, and direct targeting of TNNI3K by miR-223 were explored in this study.

\section{Materials and Methods}

Animals

One to two days old neonatal Sprague-Dawley rats were used to isolate CMs for this study. Adult rats (250g-300g, without gender restriction) were used for TAC surgery. Animals used in these experiments were 


\section{Cellular Physiology Cell Physiol Biochem 2015;35:1546-1556

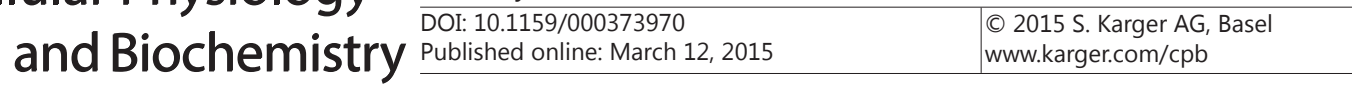

handled in accordance with the Guiding Principles in the Care and Use of Laboratory Animals, approved by Animal Care and Use Committee.

CMs culture

As we described previously [17], neonatal rat were anesthetized and sacrificed by immersing in $70 \%$ alcohol. The ventricles were removed and washed three times in warm PBS solution, then minced and incubated with $0.25 \%$ trypsinase. Dulbecco's modified Eagle's medium (DMEM, Gibco, CA, USA) containing $10 \%$ FBS (HyClone, UT, USA) was used to terminate the digestion. Digestion step was repeated four times. Cells in the supernatant were isolated by centrifugation for $10 \mathrm{~min}$ at $1500 \mathrm{rpm}$ at room temperature. CMs were cultured as monolayer at $5 \times 10^{4}$ cells $/ \mathrm{cm}^{2}$ and incubated with $\mathrm{BrdU}(0.1 \mathrm{mmol} / \mathrm{L})$ for $24 \mathrm{~h}$. Then the culture medium was changed, and cells were used for experiment.

\section{Pressure overload rat model}

Pressure overload model was created by TAC surgery. Rats were anesthetized with pentobarbital sodium $(50 \mathrm{mg} / \mathrm{kg}$, i.p.) and the suprarenal area of the descending aorta was isolated through a midline laparotomy. Then, abdominal aortic constriction surgery was performed around the abdominal aorta between the renal and superior mesenteric arteries. The sham-operated rat underwent the same surgical procedures without aorta constriction. After surgery, animals were placed in warm environment and remained connected with a rodent respirator until restoration of spontaneous breathing. Four weeks after TAC, the survived animals were sacrificed, and heart hypertrophy was confirmed by increased heart weight (HW) / body weight (BW) and left ventricular weight (LVW) / body weight (BW) ratios. Heart tissue was rapidly frozen in liquid nitrogen and stored in $-80^{\circ} \mathrm{C}$ freezer for subsequent examinations.

\section{Real-time PCR}

Total RNA was isolated from cells or tissue by using Trizol reagent (Sigma, MO, USA). Expression of miR-223 was evaluated using the mirVana ${ }^{\mathrm{TM}}$ qRT-PCR miRNA Detection Kit (Ambion, CA, USA). Primer sets for miR-223 were purchased from Applied Biosystems (CA, USA). cDNA was synthesized from $2 \mu \mathrm{g}$ total RNA (SuperScript II, GIBCO, CA, USA). Specific primers for ANP, $\alpha$-actinin, Myh6, Myh7 were listed in Table 1. GAPDH was set as internal control. Gene transcript levels were measured using SYBR green quantitative real-time PCR. Electrophoresis of reverse transcription PCR products was used as evidence for effectiveness of primers. BioEasy ${ }^{\mathrm{TM}}$ SYBR Green I kit (Bioer, Hangzhou, China) was used for quantitative measure. Reactions were prepared in triplicate for each of the two biological replicates, and the conditions were set as: $50^{\circ} \mathrm{C}$ for $2 \mathrm{~min} ; 95^{\circ} \mathrm{C}$ for $10 \mathrm{~min} ; 45$ cycles of $95^{\circ} \mathrm{C}$ for $2 \mathrm{~min}, 62^{\circ} \mathrm{C}$ for $30 \mathrm{~s}$, and $72^{\circ} \mathrm{C}$ for $30 \mathrm{~s}$. The relative quantitative expression was determined using method $2^{-\Delta \Delta c t}$.

\section{CMs hypertrophy induction and cell size measurement}

CMs were incubated in the presence or absence of the hypertrophic stimulus endothelin-1 (ET1, Sigma, MO, USA, $60 \mathrm{nmol} / \mathrm{L}$ ). Cell size was measured via surface area calculation under fluorescence microscopy after anti- $\alpha$-actinin (Sigma, MO, USA) staining. Briefly, cells on glass coverslips were washed in PBS and 4\% formaldehyde fixed at room temperature. Slide glasses were treated with $10 \%$ goat serum for $1 \mathrm{~h}$ at room temperature. Samples were stained with anti- $\alpha$-actinin $(1: 1000)$ overnight at $4^{\circ} \mathrm{C}$. After being washed in PBS, samples were incubated with fluorescein-labeled IgG and DAPI. Images were analyzed using

Table 1. Primers for RT-PCR detection of cardiac hypertrophy related marker genes

\begin{tabular}{cccc}
\hline mRNA & \multicolumn{1}{c}{ Primers $\left(5^{\prime}-3^{\prime}\right)$} & Annealing temperature $\left({ }^{\circ} \mathrm{C}\right)$ & Expected product length $(\mathrm{bp})$ \\
\hline ANP & $\begin{array}{l}\text { F: AGAAACCAGAGAGTGAGCCG } \\
\text { R: GGTGGTCTAGCAGGTTCTTGAAA }\end{array}$ & 55 & 195 \\
a- actinin & F: GGCTGTGTTCCCATCCATCGT \\
R: CCCGGTTAGCTTTGGGGTTCA & 57 & 266 \\
Myh6 & $\begin{array}{l}\text { F: AGAGTGACAGGATGACGGAT } \\
\text { R: TCCTTCACAGTCACCGTCTTG }\end{array}$ & 57 & 229 \\
Myh7 & $\begin{array}{l}\text { F: CAGTCATGGCGGATCGAGAG } \\
\text { R: CGGATTCTCCGGTGATGAGG }\end{array}$ & 54 & \\
\hline
\end{tabular}




\section{Cellular Physiology Cell Physiol Biochem 2015;35:1546-1556

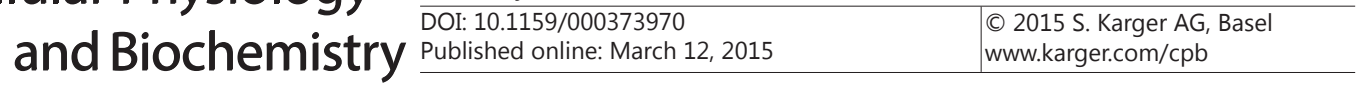 \\ Wang et al.: MicroRNA-223 Againsts Cardiomyocyte Hypertrophy by Targeting TNNI3K}

image analysis software (NIS-Elements BR, Nikon, JPN), with a photographed scale of the 10× objective lens for calibration. For each group, 50 individual CMs were measured and average value was calculated $\left(\mu \mathrm{m}^{2}\right)$, and 6 measurements in different field of microscope were performed.

\section{MiR-223 mimics transfection}

Chemically modified sense RNA (miR-223 mimic) was synthesized by Qiagen (Germany). The sequence was 5 - CGUGUAUUUGACAAGCUGAGUUG - 3 (Syn-rno-miR-223). Transfection with the miR-223 mimics was performed using HiPerFect Transfection Reagent (Qiagen, DEU). Briefly, $5 \mathrm{nM}$ of miR-223 mimics was mixed with $20 \mu \mathrm{L}$ HiPerFect in $100 \mu \mathrm{L}$ serum-free culture medium for $10 \mathrm{~min}$ at room temperature to form transfection complexes. The cells were incubated with the transfection complexes for $48 \mathrm{~h}$. Scrambled miRNA (scr-miR) that commercially synthesized by Qiagen was used as control.

\section{Recombinant Adenovirus and adenoviral infection}

Adenovirus encoding full-length rat TNNI3K (Ad.rTNNI3K) was constructed by using AdEasy Adenoviral Vector System (Agilent Technologies, CA, USA). NCBI reference sequence of TNNI3K is NM_181769.1. For adenoviral infection, cardiomyocytes were incubated for 2 hours with Ad.rTNNI3K or Ad.Vector at an approximate multiplicity of infection of 100.48 hours following infection, $95 \%$ of the cells were GFP positive.

\section{Western Blots}

Total protein was extracted from cells and heart tissue in lysis buffer for $1 \mathrm{~h}$ on ice. After centrifugation at $10000 \times \mathrm{g}$ for $5 \mathrm{~min}$, the supernatant was collected and protein concentration was determined using a Bio-Rad DC Protein Assay kit (BIORAD, CA, USA). Subsequently, 50 $\mathrm{g}$ of lysate was fractionated through a $4 \%$ stacking and 10\% running SDS-PAGE gel, and the fractionated proteins were transferred to PVDF membrane. Blots were blocked for $1 \mathrm{~h}$ at room temperature with blocking buffer. The antibodies (rabbit anti-TNNI3K, mouse anti-p-TroponinI, rabbit anti-TroponinI, mouse anti-GAPDH, SANTA CRUZ, CA, USA) were reacted with the blots overnight at $4{ }^{\circ} \mathrm{C}$. After washing $(3 \times 5 \mathrm{~min}$ in $1 \times \mathrm{TBS}-0.1 \%$ Tween 20$)$, the blots were incubated with horseradish peroxidase-conjugated secondary antibody at 1:2000 dilution for $1 \mathrm{~h}$ at room temperature. Visualization was performed with enhanced chemiluminescence. For quantification, densitometry of the target bands was divided by the corresponding densitometry of the GAPDH band using graph software (Image J 2.1.7.4, NIH, USA).

\section{Luciferase reporter assay}

To confirm that TNNI3K was targeted by miR-223, we constructed a chimeric luciferase reporter system (pMIR-REPORT, Ambion, CA, USA) tagged with the full-length $3^{\prime}$-UTR region of rat TNNI3K harboring the seedmatch sequence with ( $3^{\prime}$-mUTR, CT $\rightarrow$ GA, position 41 to 42 .) or without two nucleotide mutations ( $3^{\prime}$ UTR). HEK 293 cells were transfected with $200 \mathrm{ng}$ of pMIR-REPORT and $8 \mathrm{ng}$ of pRL-SV40 renilla luciferase control vector in the presence of the miR-223 mimics or scr-miR (as negative control) using Lipofectamine 2000 (Invitrogen, NY, USA). Luciferase activity was assayed after $48 \mathrm{~h}$ and expressed as a percentage of the luciferase activity of the vector group.

\section{Measurement of intracellular calcium}

CMs were incubated with Furo-2 (Fanbo Biochemicals, Beijing, PR China) for 30 minutes at $37^{\circ} \mathrm{C}$, to measure $\mathrm{Ca}^{2+}$ concentrations. Detection parameters were set as: excitation at $338 \mathrm{~nm}$, emission at $510 \mathrm{~nm}$. CMs were washed twice with phosphate-buffered saline (PBS), and fluorescence intensity was measured using a spectra Max M5 plate reader. Fluorescence intensity was normalized against control (Furo-2-loaded cells) after subtraction of baseline (Furo-2 without cells).

\section{Measurement of cardiomyocyte contractility}

CMs contractility was measured by using video-based edge detection system (Ion Optix Co., NY, USA). Under microscope, CMs were superfused with low flow $(1.8 \mathrm{~mL} / \mathrm{min})$ of Tyrode solution containing $\mathrm{Ca}^{2+}(1.8$ $\mathrm{mM}$ ), and electrically stimulated at $1 \mathrm{~Hz}$ at room temperature. Cell length was monitored from the brightfield image by optical edge-tracking method. The contraction amplitude was measured as the percentage of shortening of cell length. 


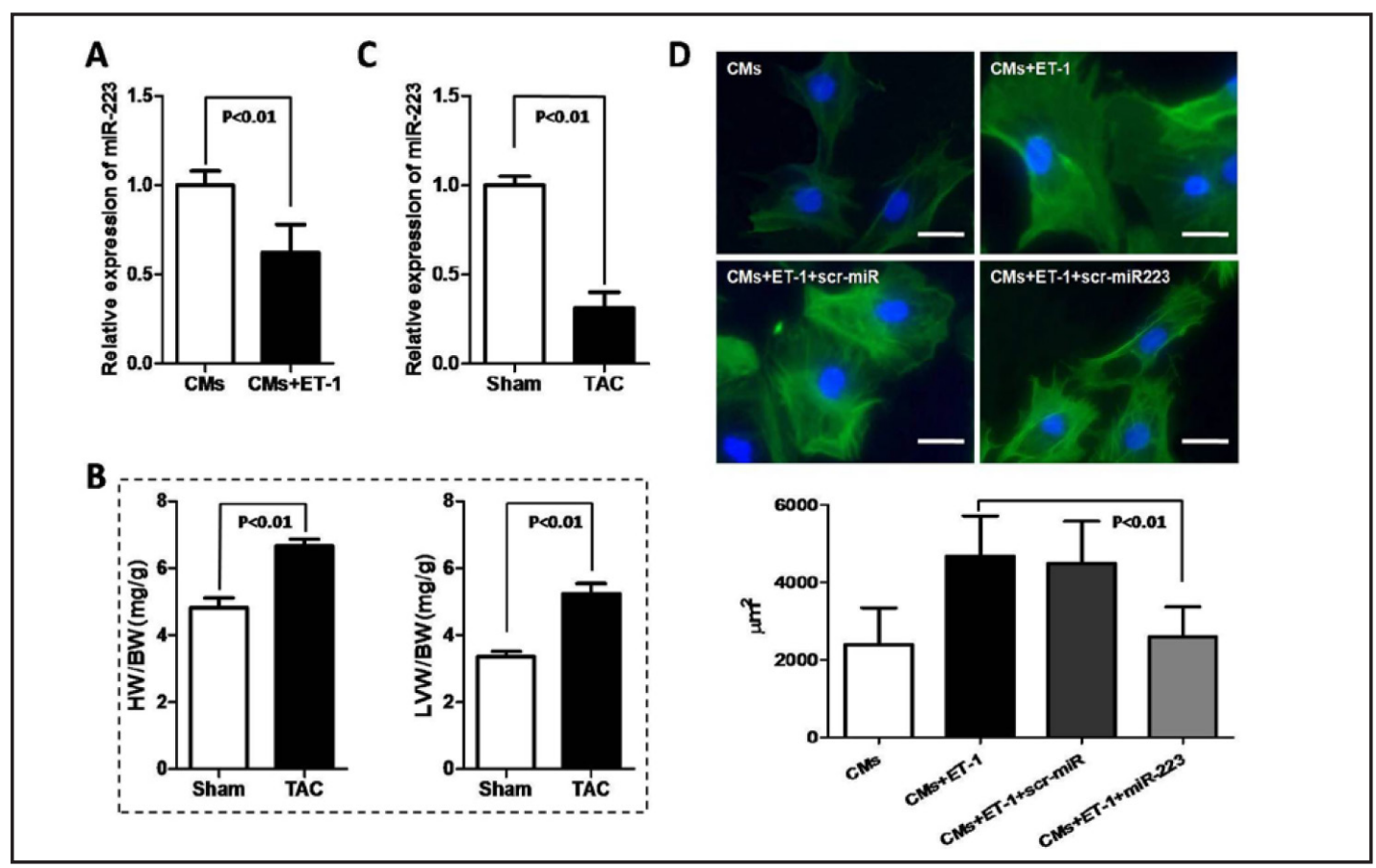

Fig. 1. Relationship of miR-223 and cardiomyocyte hypertrophy. A: In hypertrophic CMs which induced by ET-1, expression of miR-223 was measured by using real-time PCR analysis (n=6). B: Comparison of HW/ BW and LVW/BW. Sham $(n=6) ;$ TAC $(n=5)$. C: In heart tissue of TAC and Sham group, expression of miR223 was measured by using of real-time PCR analysis $(n=6)$. D: Cell size was measured via surface area calculation after anti-alpha-actinin staining (green), under fluorescence microscopy. The nucleus was stained with DAPI (blue). The figure shows a representative stain. Scale bar shows $20 \mu \mathrm{m}$. src-miR shown no effect on change of cell size in ET-1 induced hypertrophic CMs. Data are expressed as mean \pm SD for 6 individual measurements.

\section{Statistical Analysis}

Data were provided as means $\pm \mathrm{SD}$; " $n$ " represents the number of independent experiments. All data were tested for significance using ANOVA or Student's t-test as indicated. $\mathrm{P}<0.05$ was considered statistically significant.

\section{Results}

Relationship of miR-223 and cardiomyocyte hypertrophy

Expression of miR-223 in normal CMs and ET-1 induced hypertrophic CMs was detected by real-time PCR. MiR-223 mRNA expression was significantly downregulated (about $40 \%$ reduction) in hypertrophic CMs compared with control group (Fig. 1A). Accordingly, significantly downregulated miR-223 mRNA expression (about 60\% reduction) was evidenced in myocardial tissue of TAC rats compared with sham rats (Fig. 1.B and C). As shown via surface area calculation after anti- $\alpha$-actinin staining (Fig. 1D), overexpression of miR-223 in CMSs by miR-223 mimics transfection reduced ET-1 induced CMs size enlargement. These results revealed that there was a negative correlation between miR-223 expression and cardiomyocyte hypertrophy, and miR-223 is an effective regulator against cardiomyocyte hypertrophy.

Effects of miR-223 overexpression on hypertrophy marker genes in CMS

To clarify the effects of miR-223 on CMs hypertrophy, the expression levels of classic hypertrophy marker genes such as ANP, $\alpha$-actinin, Myh6, Myh7 were measured in CMs with 
Fig. 2. Assessment of hypertrophy marker genes in CMs with miR-223 mimics transfection. Relative quantitative expression of ANP, $\alpha$-actinin, Myh6, Myh7 were detected by Real-time PCR analysis (Bar graphs, $\mathrm{n}=6$ ). Electrophoresis bands demonstrated products of reverse transcription PCR which confirm the validity of PCR primers.
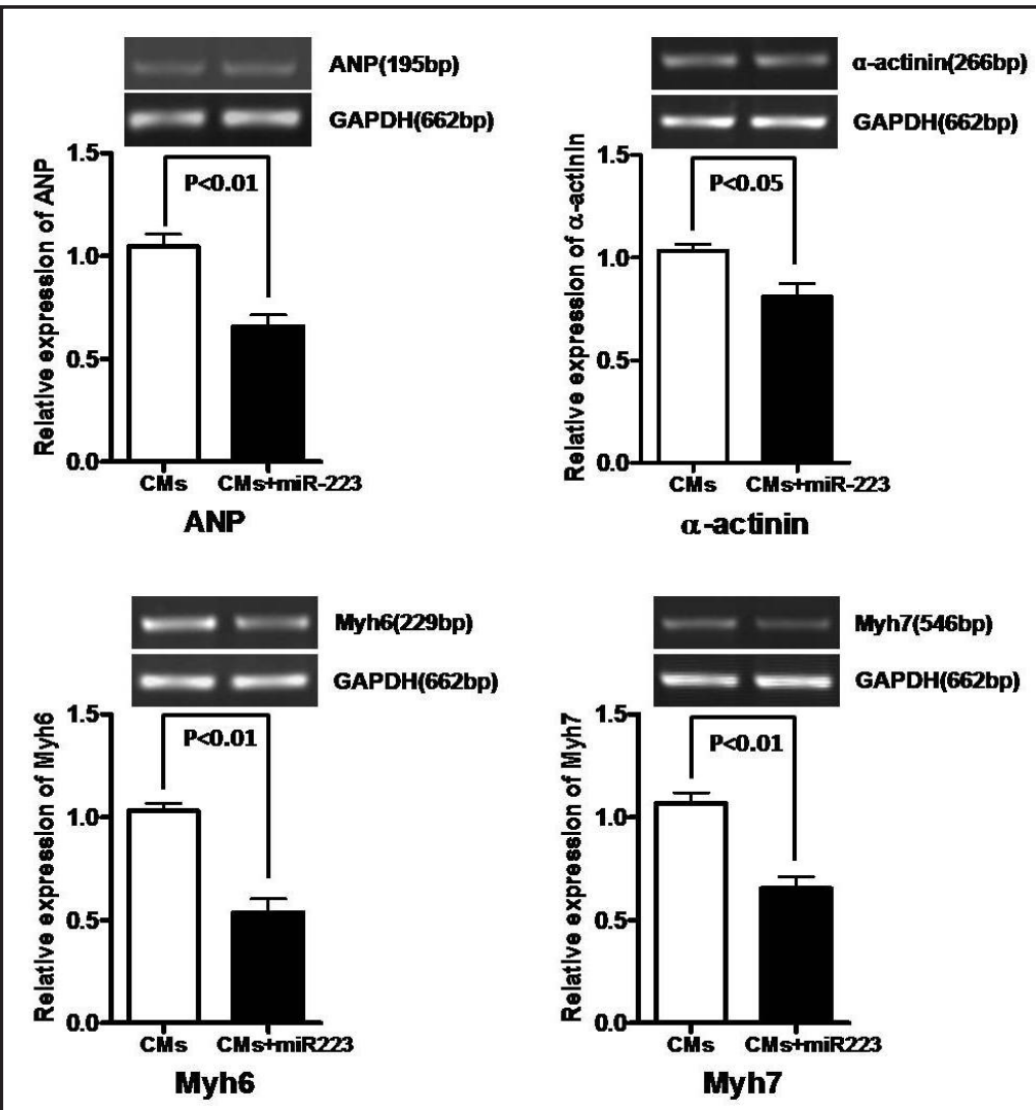

or without miR-223 mimics transfection by real-time PCR analysis. As presented in Fig. 2, expression of ANP, $\alpha$-actinin, Myh6, Myh7 were all significantly down-regulated by miR-223 (about 40\%, 20\%, 50\%, 40\% reduction, respectively). These results further supported an important role of miR-223 as a negative regulator of CMs hypertrophy.

Direct targeting of TNNI3K by miR-223 mimics transfection in CMS

Bioinformatics approach was employed to predict mRNA targets of miR-223 (www. targetscan.org). We screened rat genes that predicted to be potential targets of miR-223, narrowed them down to a few targets involved in CMs remolding, TNNI3K appeared to be a major candidate because the miR-223 seed sequence is reverse complementary to the seedmatch sequence in the 3'-UTR region of TNNI3K (Fig. 3A). As shown in Fig. 3A, luciferase activity was significantly repressed by the miR-223 mimics ( $\sim 60 \%$ reduction). This result thus provided solid evidence of the direct binding of miR-223 to the 3'-UTR of TNNI3K.

Furthermore, Western blotting was performed to examine the TNNI3K protein expression in CMs with or without miR-223 overexpression. The miR-223 mimics also induced a significant $(\sim 90 \%)$ TNNI3K downregulation of compared to the scr-miR control (Fig. 3B).

Western blotting results further confirmed the regulative link between miR-223 and TNNI3K in hypertrophic CMs, in that there was an about 2 folds higher TNNI3K protein expression in ET-1 induced hypertrophic CMs compared to control CMs, and miR-223 mimics transfection again significantly attenuated the up-regulated TNNI3K expression in ET-1 treated CMs (Fig. 3C).

Effect of miR-223 mimics transfection on cTnI phosphorylation in CMS

As TNNI3K could directly interact with cTnI, and miR-223 is an upstream regulator of TNNI3K, we also evaluated the effect of miR-223 overexpression on cTnI phosphorylation. 


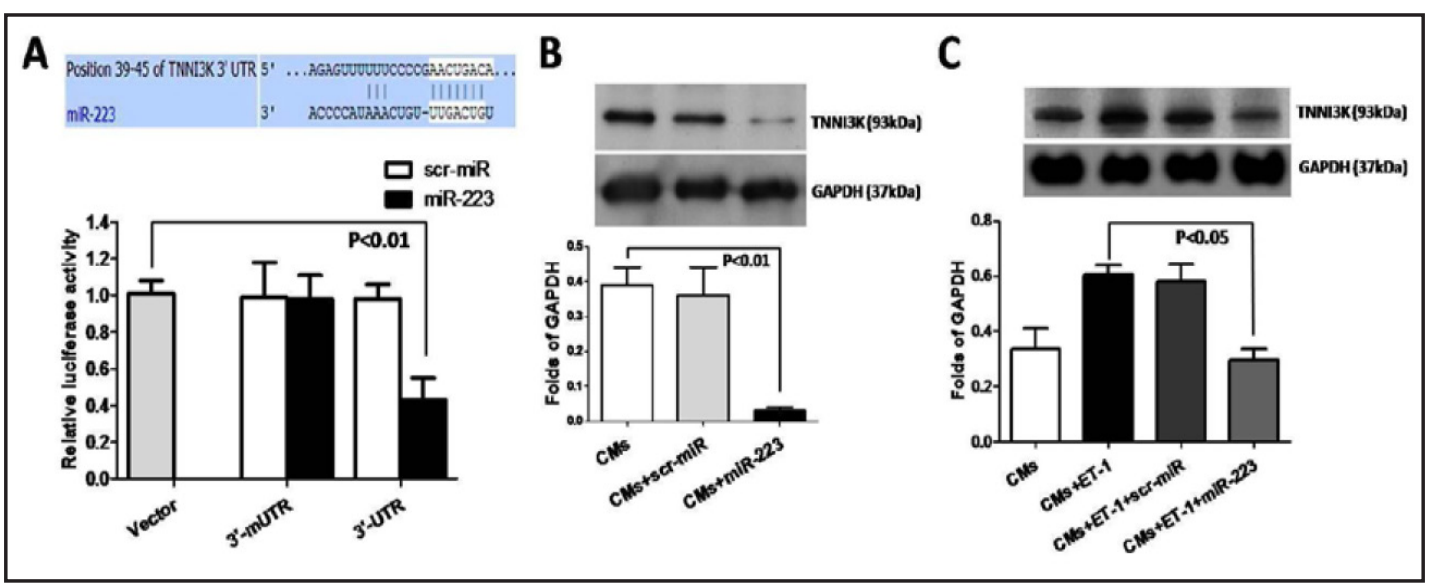

Fig. 3. Direct targeting of TNNI3K by miR-223. A: Luciferase experiment of TNNI3K 3'-UTR was conducted. The expression of the reporter containing TNNI3K 3'-UTR was suppressed by miR-223, but not in the mutated construct. src-miR exerted no effect on luciferase activity. The top blue panel showed the predicted binding site in the 3'-UTR of TNNI3K with miR-223 seed sequence. B: Western blot assessment for TNNI3K protein in CMs with miR-223 mimics transfection $(n=4)$. GAPDH was used for internal control. src-miR exerted no effect on TNNI3K expression. Immunoblots are representative of 4 independent experiments. C: Change of TNNI3K protein expression in ET-1 induced hypertrophic CMs, and the intervention effect of miR-223 mimics transfection, were detected via western blot assessment $(n=4)$. src-miR showed no effect on ET-1-enhanced TNNI3K protein expression. GAPDH was used for internal control. Immunoblots are representative of 4 independent experiments.

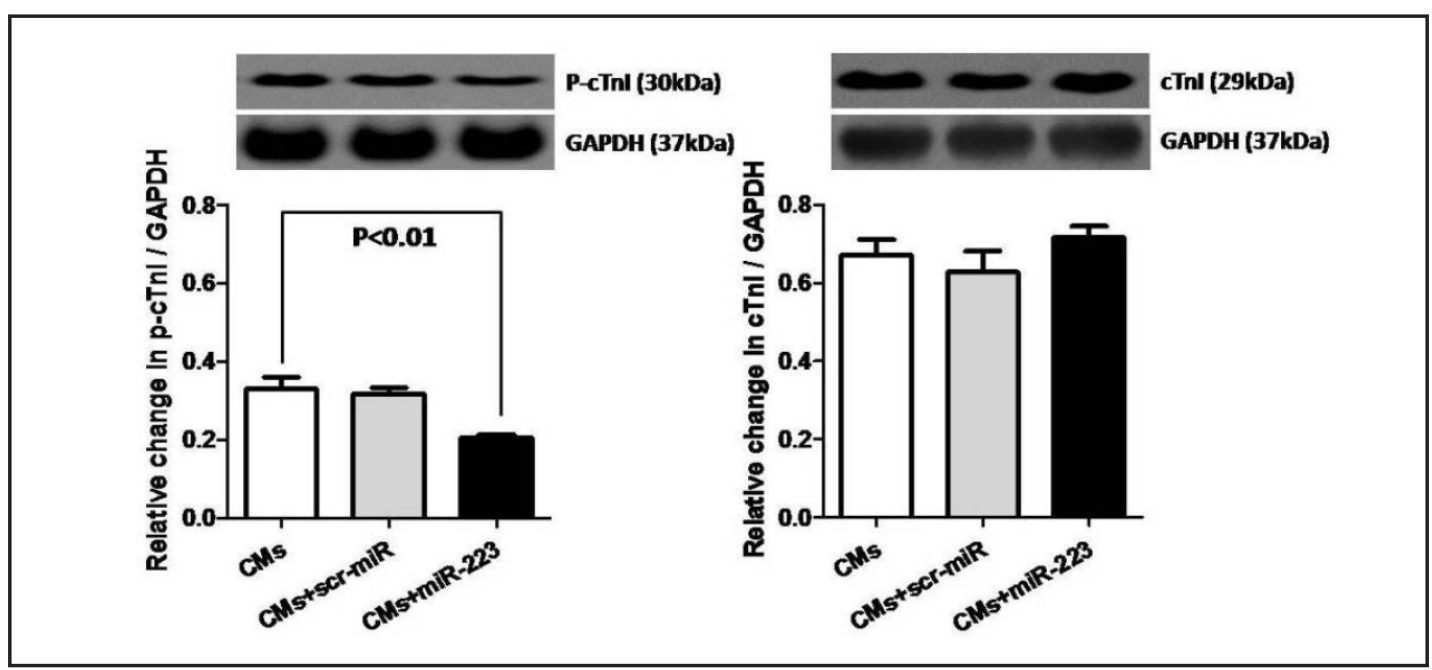

Fig. 4. miR-223 suppressed cTnI phosphorylation while did not change the expression of cTnI. Expression of phosphorylated cTnI (P-cTnI) protein was attenuated in CMs with miR-223 transfection. MiR-223 exerted no significant effect on total cTnI protein expression. There was no statistic difference between blank CMs group and src-miR group. Blots for P-cTnI protein and cTnI protein were from independent experiments. Immunoblots are representative of 3 independent experiments.

Western blot analysis showed that miR-223 overexpression significantly down-regulated cTnI phosphorylation ( $\sim 40 \%$ reduction), but exerted no significant effect on total cTnI (Fig. 4).

MiR-223 reversed the effects of TNNI3K on intracellular $\mathrm{Ca}^{2+}$ and contractility of CMS

Because changes of intracellular $\mathrm{Ca}^{2+}$ and contractility of CMs are highly related to cardiomyocyte hypertrophy, we observed the impacts of miR-223 overexpression on these 


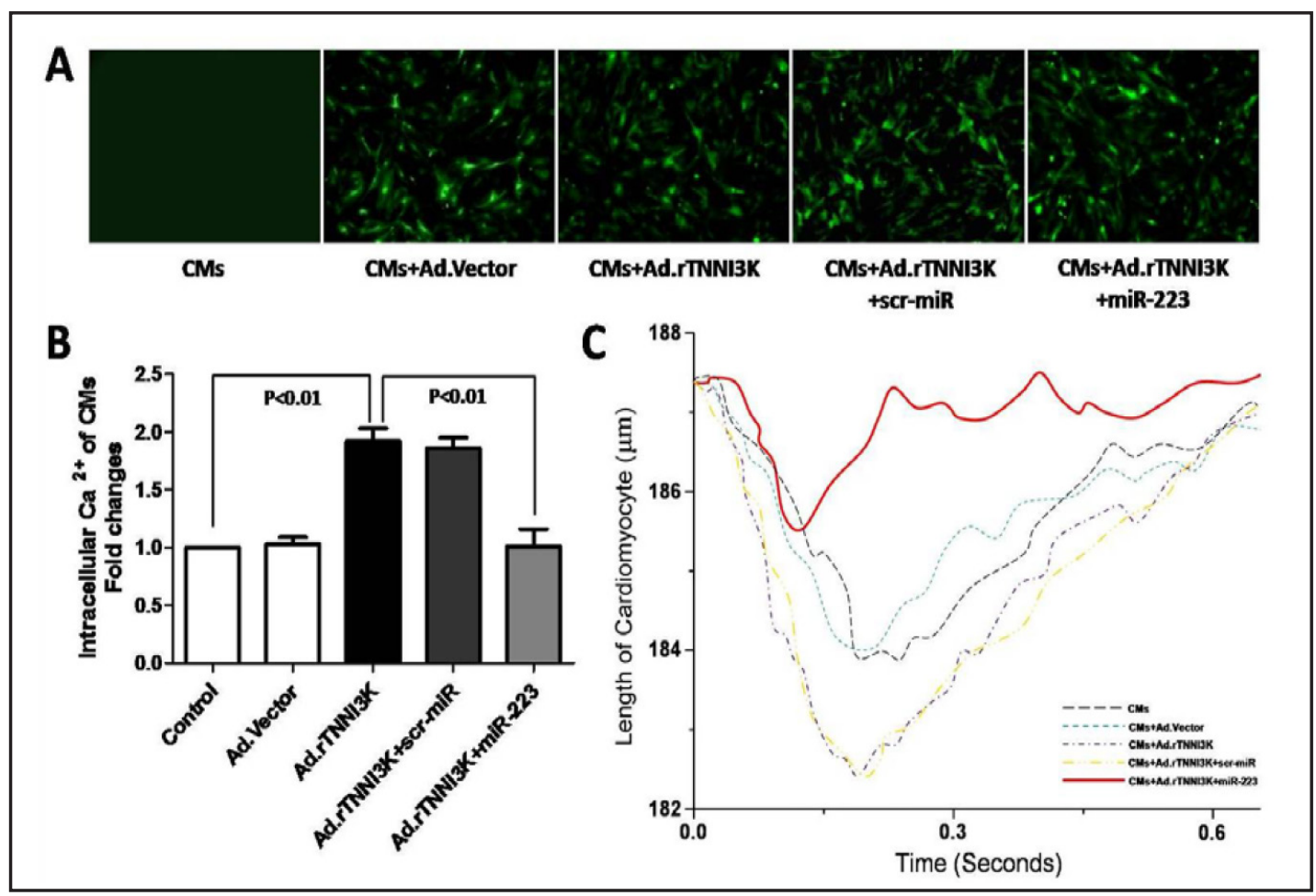

Fig. 5. miR-223 reversed the effects of TNNI3K on intracellular $\mathrm{Ca}^{2+}$ and cardiomyocyte contractility. A: Expression of recombinant adenoviral transgene in CMs. CMs were infected with adenoviral vectors carrying EGFP as marker gene, after $24 \mathrm{~h}$ infection in vitro culture (100 multiplicity), with/without miR-223 mimics or scr-miR transfection. B: Ad.rTNNI3K induced elevation of intracellular $\mathrm{Ca}^{2+}$ concentrations in CMs. miR223 reversed the enhanced intracellular $\mathrm{Ca}^{2+}$. The quantification of intracellular $\mathrm{Ca}^{2+}$ level was measured by using Furo-2 dye staining $(n=6)$. There was no statistic difference between Ad.rTNNI3K group and Ad.rTNNI3K+src-miR group. C: Effects of miR-223 / TNNI3K on cardiomyocyte contraction. Representative traces of cell shortening from non-infected CMs, CMs infected with Ad.Vector or Ad.rTNNI3K, and Ad.rTNNI3K transgenic CMs with miR-223 mimics or scr-miR transfection were recorded. Ad.Vector and src-miR exerted no effect on cardiomyocyte contraction.

cellular characteristics of CMs. Changes on Ad.rTNNI3K (Ad.Vector as vector control) infected CMs, with or without miR-223 mimics co-transfection (scr-miR as control) were evaluated (Fig. 5A). Ad.rTNNI3K induced an about $90 \%$ increase in intracellular $\mathrm{Ca}^{2+}$ concentrations in CMs, while this effect could be significantly blocked by miR-223 mimics co-transfection (Fig. 5B). As shown in Fig. 5C, Ad.rTNNI3K induced a significant increase in cell shortening of CMs (Ad.rTNNI3K group: $2.69 \pm 0.11 \%$ vs. blank control group: $1.81 \pm 0.06 \%, \mathrm{p}<0.05$ ) while miR223 mimics significantly blocked this effect (Ad.rTNNI3K plus miR-223 group: $0.98 \pm 0.08 \%$ vs. blank control group, $\mathrm{p}<0.01$ ).

\section{Discussion}

Heart undergoes compensatory hypertrophic growth and cardiac remodeling for sustaining cardiac output and these processes will sometimes result in cardiac dysfunction in the long-term [18]. The process of pathological myocardial hypertrophy is a highly complex event, and has been studied in relation to miRNAs to date. Whole arrays in pathological hypertrophy animal models indicated that some particular miRNAs were upregulated or downregulated, such as miR-1, miR-18b, miR-21, miR-133, miR-208 [19-21]. Present study showed that change in endogenous miR-223 expression was related to cardiac hypertrophy, and overexpression of miR-223 by miR-223 mimics transfection reduced the ET- 1 induced 
hypertrophic response in neonatal rat CMs. MiR-223 overexpression also suppressed activation of hypertrophic marker genes (ANP, $\alpha$-actinin, Myh6, Myh7). These results revealed that miR-223 appears to be a negative regulator of cardiac hypertrophy.

CMs contain arrays of serially aligned and laterally registered sarcomeres that parallel the longitudinal cellular axis. Some specific proteins comprise the sarcomere's thick myofilament, intermediate myofilament, and thin myofilament [22]. The mechanism for cardiac hypertrophy is related to changes in the organization of the sarcomeric structure that induced by altered protein phosphorylation [23]. There is evidence that CMs hypertrophy is provoked by the activation of protein kinase cascades which, in turn, activate MAPK. These kinases are serine-threonine kinases and phosphorylate a number of important cellular substrates for cell growth and differentiation processes that related to cardiac hypertrophy [24]. In recent years, the contribution of some novel protein kinases involved in cardiac hypertrophy has been the subject of fairly detailed study. TNNI3K, as a cardiomyocytespecific kinase, is known to promote ischemia/reperfusion injury, oxidative stress, and CMs death. TNNI3K-mediated injury is mediated through increased mitochondrial superoxide production and impaired mitochondrial function and is largely dependent on p38 mitogenactivated protein kinase (MAPK) activation [25]. Recent studies have revealed that TNNI3K is associated with hemodynamic overload induced cardiac remodeling. TNNI3K overexpression readily induced CMs hypertrophy and accelerated hypertrophy in hypertrophic CMs [15]. By using bioinformatics approach, we found TNNI3K as a predict target of miR-223, in fact, present study revealed that miR-223 mimics transfection obviously shut down the TNNI3K protein expression and canceled hypertrophy-induction effect of TNNI3K on CMs. Luciferase assay also confirmed the direct targeting of TNNI3K by miR-223 overexpression. These results collectively suggest an importantrole of miR-233 / TNNI3K pathway in modulating CMs hypertrophy.

It was shown that TNNI3K could promote concentric hypertrophy with enhancement of cardiac function via regulating the phosphorylation of cTnI [26] and thus contributed to the regulation of cardiac myofilament contraction [27]. Troponin I (TnI) is a known inhibitory unit of the troponin complex associated with the thin filament. It links $\mathrm{Ca}^{2+}-\mathrm{TnC}$ binding with activation of cross bridge reactions with the thin filament, and thus inhibits actomyosin interactions at diastolic levels of intracellular $\mathrm{Ca}^{2+}$. cTnI also represents a key regulatory protein in cardiac muscle contraction and relaxation [28]. In line with above findings, present study revealed the suppressive effect of miR-233/TNNI3K pathway on cTnI phosphorylation. Attention has also been focused on changes in the intracelluer $\mathrm{Ca}^{2+}$ cycle of CMs, and the contractual capacities of CMs. It is widely accepted that the increase of intracellular $\mathrm{Ca}^{2+}$ could activate hypertrophic program. Our results demonstrated the TNNI3K induced elevation of the $\mathrm{Ca}^{2+}$ concentration in CMs, and this effect could be abrogated by miR-223 mimic transfection. In addition, the contractility of individual ventricular CMs was enhanced by TNNI3K overexpression, while suppressed by miR-223 mimics. These observations supplied evidence of the modulating role miR-233 / TNNI3K pathway oncTnI phosphorylation and CMs hypertrophy.

It's worth noting that early data suggested TNNI3K had a protective role post myocardial injury, based on findings of increased beating mass and number of $\alpha$-actinin-positive cells after TNNI3K overexpression [13]. We think these observed results might actually supporte the "myogenetic" but not "protective" function of TNNI3K, and in some ways, were consistent with pro-hypertrophic effect of TNNI3K observed in present study. Indeed, most of recent studies demonstrated that both normal expression and TNNI3K overexpression presented a detrimental effect on cardiac function [14, 15, 25]. Our experimental data also suggested the detrimental pro-hypertrophic role of TNNI3K, and provided a preliminary exploration on TNNI3K's upstream regulatory mechanism. However, further studies are warranted to explore the exact function and mechanism of TNNI3K in regulating cardiac remodeling and function.

In summary, we show that, as negative regulator to CMs hypertrophy, miR-223 expression is downregulated in ET-1 induced CMs and in myocardial tissue of TAC rats 


\section{Cellular Physiology Cell Physiol Biochem 2015;35:1546-1556 \begin{tabular}{l|l} 
and Biochemistry 1159/000373970 & $\begin{array}{l}\text { O 2015 S. Karger AG, Basel } \\
\text { www.karger.com/cpb }\end{array}$ \\
\hline
\end{tabular}

while miR-223 overexpression can suppress ET-1 induced CMs hypertrophy. By direct targeting TNNI3K, miR-223mimic transfection could downregulate cTnI phosphorylation, and reverse the effects of TNNI3K on intracellular $\mathrm{Ca}^{2+}$ and contractility of CMs. Therefore, miR-223 appears to be a promising candidate for inhibiting CMs hypertrophy, and miR-223 / TNNI3K axis might be one of interesting targets for the clinical treatment of hypertrophy.

\section{Acknowledgments}

This work was supported by Young Scholars Fund from Shanghai Municipal Commission of Health and Family Planning, No. 2013Y068; Jiangxi Science Fund for Young Scholars, No. 20114BAB215015; Grant from XinHua Hospital affiliated to Shanghai Jiao Tong University School of Medicine, No. 13YJ16.

We thank for the technology supports from HaoBo Biological Technology.LTD (Shanghai, PR China). We also thank for advices in manuscript writing given by Dr. Shuhong Li from Toronto General Hospital (Ontario, Canada). We thank Dr. Kai Hu from Würzburg University for his help in the preparation of present manuscript.

\section{References}

1 Oka T, Akazawa H, Naito AT, Komuro I: Angiogenesis and cardiac hypertrophy: maintenance of cardiac function and causative roles in heart failure. Circ Res 2014;114:565-571.

2 Di J, Jiang L, Zhou Y, Cao H, Fang L, Wen P, Li X, Dai C, Yang J: Ets-1 targeted by microRNA-221 regulates angiotensin II-induced renal fibroblast activation and fibrosis. Cell Physiol Biochem 2014;34:1063-1074.

3 Wang J, Yang X: The function of miRNA in cardiac hypertrophy. Cell Mol Life Sci 2012;69:3561-3570.

4 Zhou S, Liu Y, Prater K, Zheng Y, Cai L: Roles of microRNAs in pressure overload-and ischemia-related myocardial remodeling. Life Sci 2013;93:855-862.

5 Johnnidis JB, Harris MH, Wheeler RT, Stehling-Sun S, Lam MH, Kirak O, Brummelkamp TR, Fleming MD, Camargo FD. Regulation of progenitor cell proliferation and granulocyte function by microRNA-223. Nature 2008;451:1125-1129.

6 Ma L, Chen Y, Zhang B, Liu G: Increased microRNA-223 in Helicobacter pylori-associated gastric cancer contributed to cancer cell proliferation and migration. Biosci Biotechnol Biochem 2014;78:602-608.

7 Song L, Duan P, Guo P, Li D, Li S, Xu Y, Zhou Q: Downregulation of miR-223 and miR-153 mediates mechanical stretch-stimulated proliferation of venous smooth muscle cells via activation of the insulin-like growth factor-1 receptor. Arch Biochem Biophys 2012;528:204-211.

8 Pan Y, Meng M, Zhang G, Han H, Zhou Q: Oncogenic MicroRNAs in the Genesis of Leukemia and Lymphoma. Curr Pharm Des 2014;20:5260-5267.

9 Sugatani T, Hruska KA: MicroRNA-223 is a key factor in osteoclast differentiation. J Cell Biochem 2007;101:996-999.

10 Lu H, Buchan RJ, Cook SA. MicroRNA-223 regulates Glut4 expression and cardiomyocyte glucose metabolism. Cardiovasc Res 2010;86:410-4120.

11 Dickinson BA, Semus HM, Montgomery RL, Stack C, Latimer PA, Lewton SM, Lynch JM, Hullinger TG, Seto AG, van Rooij E. Plasma microRNAs serve as biomarkers of therapeutic efficacy and disease progression in hypertension-induced heart failure. Eur J Heart Fail 2013;15:650-659.

12 Ritchie W, Rasko JE, Flamant S: MicroRNA target prediction and validation. Adv Exp Med Biol 2013;774:3953.

13 Lai ZF: TNNI3K could be a novel molecular target for the treatment of cardiac diseases. Recent Pat Cardiovasc Drug Discov 2009; 4:203-210.

14 Tang H, Xiao K, Mao L, Rockman HA, Marchuk DA: Overexpression of TNNI3K, a cardiac-specific MAPKKK, promotes cardiac dysfunction. J Mol Cell Cardiol 2013;54:101-111. 


\section{Cellular Physiology Cell Physiol Biochem 2015;35:1546-1556 and Biochemistry

15 Wang L, Wang H, Ye J, Xu RX, Song L, Shi N, Zhang YW, Chen X, Meng XM: Adenovirus-mediated overexpression of cardiac troponin I-interacting kinase promotes cardiomyocyte hypertrophy. Clin Exp Pharmacol Physiol 2011;38:278-284.

16 Funayama A, Shishido T, Netsu S, Narumi T, Kadowaki S, Takahashi H, Miyamoto T, Watanabe T, Woo CH, Abe J, Kuwahara K, Nakao K, Takeishi Y, Kubota I: Cardiac nuclear high mobility group box 1 prevents the development of cardiac hypertrophy and heart failure. Cardiovasc Res 2013;99:657-664.

17 Wang YS, Zhou J, Liang C, Hong K, Cheng XS, Wu ZG. ERK5 knock down aggravates detrimental effects of hypothermal stimulation on cardiomyocytes via Bim upregulation. Environ Toxicol Pharmacol 2013;36:724-731.

18 Mann DL. Basic mechanisms of left ventricular remodeling: the contribution of wall stress. J Card Fail 2004;10:S202-206.

19 Sayed D, Hong C, Chen IY, Lypowy J, Abdellatif M: MicroRNAs play an essential role in the development of cardiac hypertrophy. Circ Res 2007;100:416-424.

20 Kuppusamy KT, Sperber H, Ruohola-Baker H: MicroRNA regulation and role in stem cell maintenance, cardiac differentiation and hypertrophy. Curr Mol Med 2013;13:757-764.

21 Martinelli NC, Cohen CR, Santos KG, Castro MA, Biolo A, Frick L, Silvello D, Lopes A, Schneider S, Andrades ME, Clausell N, Matte U, Rohde LE: An Analysis of the Global Expression of MicroRNAs in an Experimental Model of Physiological Left Ventricular Hypertrophy. PLoS One 2014;9:e93271.

22 Landstrom AP, Ackerman MJ. Beyond the cardiac myofilament: hypertrophic cardiomyopathy-associated mutations in genes that encode calcium-handling proteins. Curr Mol Med 2012; 12: 507-18.

23 Kuo PL, Lee H, Bray MA, Geisse NA, Huang YT, Adams WJ, Sheehy SP, Parker KK. Myocyte shape regulates lateral registry of sarcomeres and contractility. Am J Pathol 2012;181:2030-2037.

24 Katta A, Thulluri S, Manne ND, Addagarla HS, Arvapalli R, Nalabotu SK, Gadde M, Rice KM, Blough ER: Overload induced heat shock proteins (HSPs), MAPK and miRNA (miR-1 and miR133a) response in insulin-resistant skeletal muscle. Cell Physiol Biochem 2013;31:219-229.

25 Vagnozzi RJ, Gatto GJ Jr, Kallander LS, Hoffman NE, Mallilankaraman K, Ballard VL, Lawhorn BG, Stoy P, Philp J, Graves AP, Naito Y, Lepore JJ, Gao E, Madesh M, Force T: Inhibition of the cardiomyocyte-specific kinase TNNI3K limits oxidative stress, injury, and adverse remodeling in the ischemic heart. Sci Transl Med 2013;5:207ra141.

26 Wang X, Wang J, Su M, Wang C, Chen J, Wang H, Song L, Zou Y, Zhang L, Zhang Y, Hui R: TNNI3K, a cardiacspecific kinase, promotes physiological cardiac hypertrophy in transgenic mice. PLoS ONE 2013;8:e58570.

27 Wang H, Wang L, Song L, Zhang YW, Ye J, Xu RX, Shi N, Meng XM: TNNI3K is a novel mediator of myofilament function and phosphorylates cardiac troponin I. Braz J Med Biol Res 2013;46:128-137.

28 Layland J, Solaro RJ, Shah AM: Regulation of cardiac contractile function by troponin I phosphorylation. Cardiovasc Res 2005;66:12-21. 